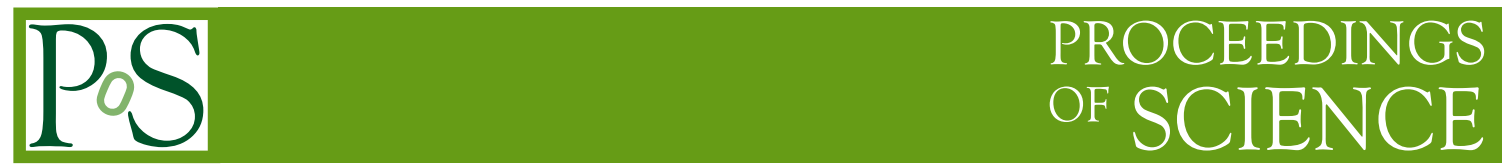

\title{
Neutral BSM Higgs searches in ATLAS
}

\section{Lei Zhang*, on behalf of ATLAS collaboration}

Fakultät für Mathematik und Physik, Albert-Ludwigs-Universität, Freiburg, Germany

E-mail: lei.zhang@cern.ch

\begin{abstract}
This report summarizes the latest results of the neutral heavy BSM Higgs boson search, mainly based on data collected by the ATLAS experiment at a center-of-mass energy of $\sqrt{s}=13 \mathrm{TeV}$ in 2015 and 2016, with an integrated luminosity of 3.2-15.4 fb $\mathrm{fb}^{-1}$. Fermionic, bosonic and di-Higgs final states have been investigated in a mass range from $200 \mathrm{GeV}$ to $3000 \mathrm{GeV}$. No sign for a new heavy neutral resonance has been found in data so far, and stringent upper limits are reported.
\end{abstract}

Prospects for Charged Higgs Discovery at Colliders

3-6 October 2016

Uppsala, Sweden

${ }^{*}$ Speaker. 


\section{Introduction}

With the Higgs boson of $125 \mathrm{GeV}$ mass discovered [1, 2], the Standard Model (SM) of particle physics was complemented by the last missing piece. However, the SM can not explain many phenomena, e.g. the Higgs boson mass hierarchy problem, neutrino masses, baryon genesis, dark matter, etc. Therefore, many Beyond SM (BSM) theories have been proposed to solve these problems. Many of these theories predict a more complex Higgs sector featuring more, usually heavier, Higgs bosons. For example, a generic BSM model in the Higgs sector, Two Higgs Doublet Model (2HDM), introduces two Higgs doublets and predicts five Higgs bosons: two neutral CP-even $h$ and $H$, one neutral CP-odd $A$, and two charged $H^{ \pm}$. The ratio of the vacuum expectation values of the two doublets is defined as $\tan \beta$. The Higgs sector of Minimum Supersymmetric Standard Model (MSSM) can be considered as one type of 2HDM models. In 2015, ATLAS started to take data at the center-of-mass energy of $\sqrt{s}=13 \mathrm{TeV}$ which is about two times higher than the energy of the previous data-taking. The increased collision energy enlarges the accessible phase space of high mass resonances. In this report, the results of the searches for a heavy neutral Higgs boson by the ATLAS experiment [3] are presented. The heavy neutral Higgs boson can couple to SM particles in a similar way as the SM Higgs boson. Therefore, all the SM Higgs boson search channels, like the decay into a pair of fermions, vector bosons or SM-like Higgs bosons, need to be exploited. Section 3 presents the searches for a heavy Higgs boson decaying into fermions. Section 4 presents the searches for a heavy Higgs boson decaying into vector bosons. Section 5 discusses the heavy Higgs boson searches in the di-Higgs final state.

\section{Status after LHC Run 1}

References $[4,5]$ summarize the results of many BSM Higgs boson searches performed by the ATLAS experiment after LHC Run 1. Exclusion limits of different searches have been interpreted consistently in several models within the extended Higgs sector. As discussed in Refs. [4, 5], Figure 1 depicts the constrained parameter space of hMSSM which is one type of MSSM model with $m_{h}$ set as $125 \mathrm{GeV}$. The different colors represent the $95 \%$ confidence level exclusion limits of different BSM Higgs boson searches. One can clearly observe that those different analyses constrain different parts of the parameter space. This originates from the differing coupling structure of the heavy Higgs boson to the up-/down-type fermions, vector bosons, and Higgs bosons in those different models. This example underlines the importance of a wide search program for new heavy Higgs boson as pursued within the ATLAS collaboration.

\section{Decays into fermions}

One of the standard search channels in the MSSM is the decay $H \rightarrow \tau \tau$, as very often downtype fermion couplings are enhanced and the di-tau final state provides a rather clean search topology. ATLAS has performed an analysis based on an integrated luminosity of about $13.3 \mathrm{fb}^{-1}$ at a center of mass energy of $\sqrt{s}=13 \mathrm{TeV}$ in Ref. [6]. The analysis is divided into two channels according to the decay modes of the tau-lepton: one leptonic tau decay plus one hadronic tau decay ( $\left.\tau_{\text {lep }} \tau_{\text {had }}\right)$ or two hadronic tau decays $\left(\tau_{\text {had }} \tau_{\text {had }}\right)$. To enhance the sensitivity to the $b$ quark associated 


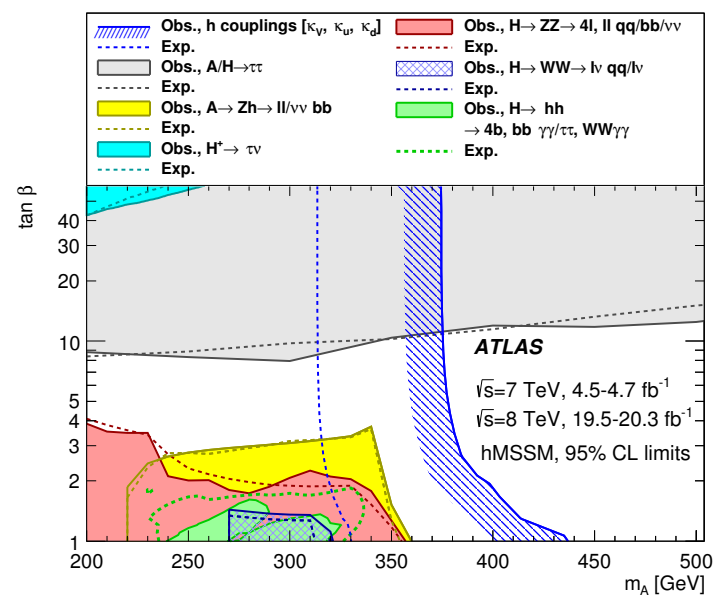

Figure 1: Run 1 summary: Regions of the $\left[\mathrm{m}_{\mathrm{A}}, \tan \beta\right]$ plane excluded in the hMSSM via direct searches for heavy Higgs bosons and fits to the measured rates of observed Higgs boson production and decay modes. Plot from Refs. [4, 5].

production mode, both channels further categorize events based on whether they have $b$-tagged jets or not. Figure 2a shows the distribution of the final discriminant variable, the total transverse mass defined in Ref. [6], in the $\tau_{\text {lep }} \tau_{\text {had }}$ channel. In the explored high mass region, no excess in the data compared to the expected background is found. Figure $2 \mathrm{~b}$ shows the exclusion limit in the hMSSM model parameter space.

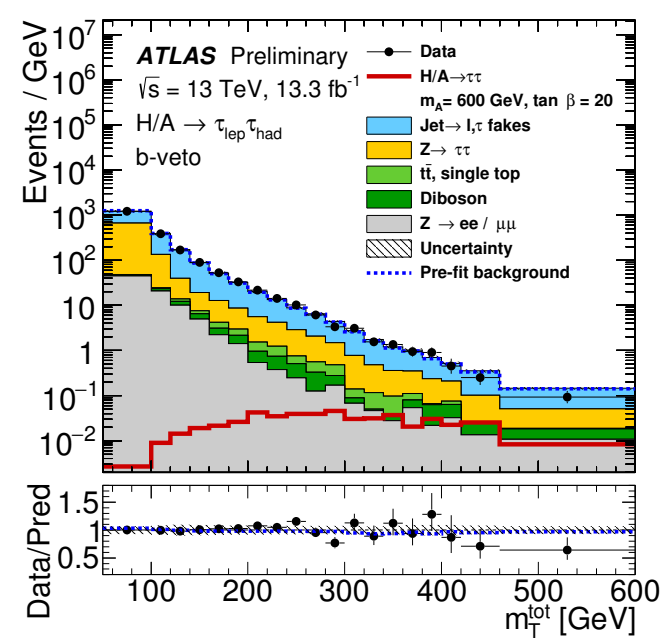

(a) Final discriminant variable distribution.

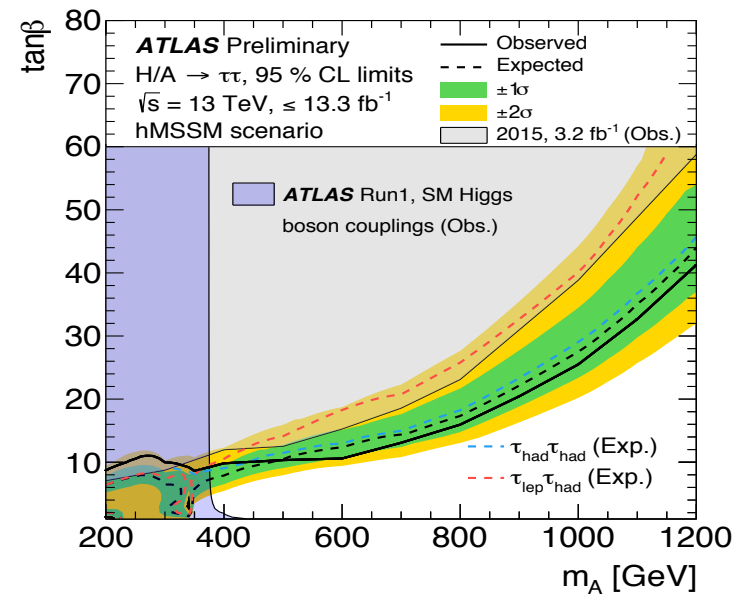

(b) Exclusion limit in the $\left[\mathrm{m}_{\mathrm{A}}, \tan \beta\right]$ plane of the hMSSM model.

Figure 2: Search for a heavy Higgs decaying into $\tau \tau$. Plots from Ref. [6].

Another important channel in the sector of searches for heavy Higgs bosons decaying fermionically is the decay into a pair of up-type fermions, e.g. top quarks. As a complement to the $\tau \tau$ channel, the $t \bar{t}$ channel is sensitive to the high mass and the low $\tan \beta$ region. With the same event selection applied as that in Ref. [7], ATLAS $8 \mathrm{TeV}$ Run 1 data are re-analyzed with the 2HDM interpretation [8]. A large effort has been spent to properly take into account the interference be- 
tween the signal and the SM $t \bar{t}$ process. The results exclude $\tan \beta<0.85$ for $\mathrm{m}_{\mathrm{A}}=500 \mathrm{GeV}$ and $\tan \beta<0.45$ for $\mathrm{m}_{\mathrm{H}}=500 \mathrm{GeV}$, while no value of $\tan \beta$ can be excluded for the second considered masses point above $750 \mathrm{GeV}$.

\section{Decays into vector bosons}

Heavy Higgs bosons decaying into vector bosons, i.e. $Z Z / W W$, are predicted by various BSM scenarios. In light of an $2.5 \sigma$ excess found around $2 \mathrm{TeV}$ in the hadronic final state by the ATLAS experiment in the LHC Run 1 [9], a search for a heavy Higgs boson decaying into $Z Z / W W$ has been performed in the final state with hadronic jets based on the data collected by ATLAS in 2015. No excess in the data compared to the expected background was found, and exclusion limits were reported [10]. This report will focus on the updated results based on data collected by ATLAS before August 2016.

Searches for a heavy Higgs boson decaying into $4 l$, via two $Z$ bosons [11] are performed based on the $13 \mathrm{TeV}$ data with an integrated luminosity of $14.8 \mathrm{fb}^{-1}$. Figure $3 \mathrm{a}$ shows the 4lepton invariant mass distribution and no sign for a heavy resonance is found. Figure $3 \mathrm{~b}$ shows the exclusion limit for an assumed signal with a narrow width for the gluon fusion (ggF) production mode. The results for other signal assumptions as well as an interpretation with an Effective Field Theory approach are reported in Ref. [11].

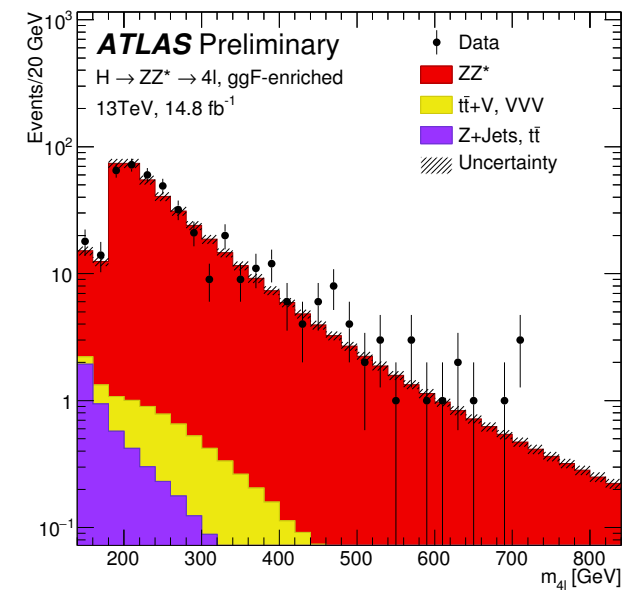

(a) $4 l$ invariant mass distribution.

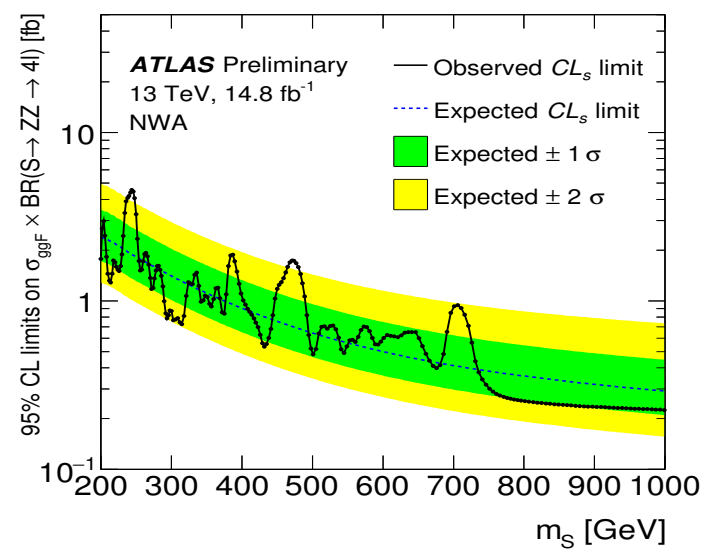

(b) Exclusion limit on cross section times branching ratio as a function of the Higgs boson mass.

Figure 3: Search for a heavy Higgs decaying into $Z Z \rightarrow 4 l$. Plots from Ref. [11].

Compared to the $4 l$ channel, $H \rightarrow Z Z$ events with a $Z \rightarrow j j$ decay have larger branching fractions, but suffer from poor energy resolution and large background from multi-jet processes. At the high explored mass region, the hadronic channel become important, as less QCD jets events can contaminate the signal region with energetic jets from $W / Z$ boson decays. Heavy Higgs decaying into $l l q q$ and $v v q q$ searches are performed by the ATLAS experiment, as reported in Ref. [12]. Both $l l q q$ and $v v q q$ channels are divided into two categories: resolved category where $Z \rightarrow q q$ is reconstructed from two jets with $R=0.4$ and merged category where $Z \rightarrow q q$ is reconstructed from one large- $R$ jet with $R=1.0$. Jet substructure techniques described in Refs. [13, 14] have 
been applied to the merged category. Figure 4a shows the $l l q q$ invariant mass distribution and no sign for a heavy resonance is found. Figure $4 \mathrm{~b}$ shows the exclusion limit for the $l l q q$ channel in the ggF production mode. The results of the $v v q q$ channel and the exclusion limit on other signal hypotheses can be found in Ref. [12].

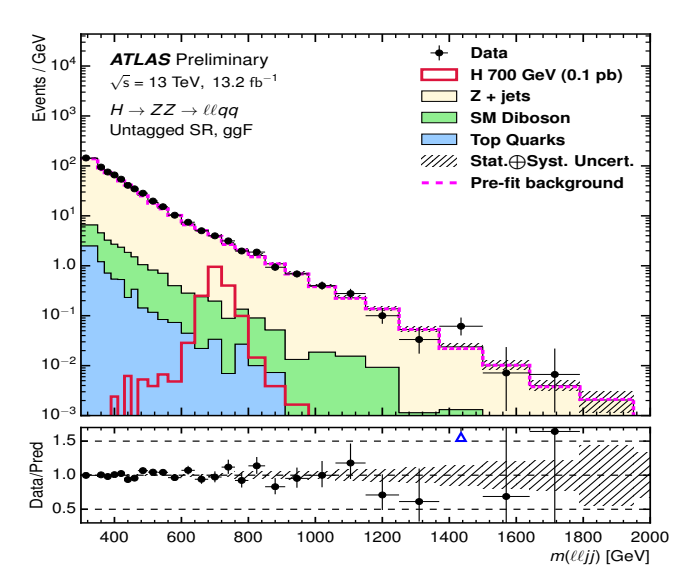

(a) $l l j j$ invariant mass.

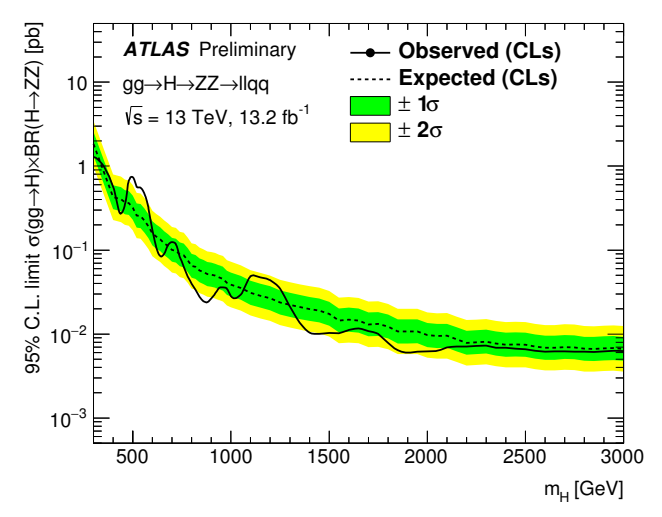

(b) Exclusion limit on cross section times branching ratio as a function of the Higgs boson mass.

Figure 4: Search for a heavy Higgs decaying into $Z Z \rightarrow l l q q$. Plots from Ref [12].

A heavy Higgs boson decaying into $W W$ has been searched in $l v q q^{\prime}[15]$ and $l v l v$ [16] channels by ATLAS. $W W \rightarrow l v q q^{\prime}$ is divided into resolved and merged categories, like $Z Z \rightarrow l l / v v q q$. For the $l v l v$ channel, in order to reduce the Drell-Yan background, only the mixed flavour channel $e v \mu v$ is used. Figure 5a shows the $l v q q^{\prime}$ invariant mass for the merged category. Figure 5b shows the transverse mass of the di-lepton and $\mathrm{E}_{\mathrm{T}}^{\mathrm{miss}}$ system.. No sign for a heavy resonance is found and exclusion limits are shown in Figure 6.

Based on data collected in 2015, ATLAS [17] and CMS [18] experiments reported a slight excess in the di-photon invariant mass spectrum. With the $15.4 \mathrm{fb}^{-1}$ newly collected data, ATLAS updated this analysis [19], as shown in Figure 7a. The excess is not confirmed and the exclusion limit is reported in Figure 7b.

\section{Decays into Higgs boson pairs}

After the discovery of the SM-like Higgs boson, with increased collision energy and integrated luminosity, searches for a heavy resonance decaying into two SM-like Higgs bosons become more relevant. ATLAS has published di-Higgs boson searches in several search channels, i.e. $H H \rightarrow$ $b b \gamma \gamma, b b \tau \tau, \gamma \gamma W W^{*}$ and $b b b b$ in Ref. [20]. As shown in Figure 8, $b b \gamma \gamma$ is the most sensitive channel in the low mass regime while $b b b b$ is the most sensitive channel in the high mass regime.

In this report, we focus on the $b b b b$ channel which has been updated recently based on the collected $13.3 \mathrm{fb}^{-1}$ of data at the $13 \mathrm{TeV}$ collision energy, as reported in Ref. [21]. Like hadronic heavy Higgs to $Z Z / W W$ search channels, the $b b b b$ analysis is also divided into resolved and merged categories. Figure 9 a shows the $4 b$ invariant mass distribution in the merged category. No sign for a heavy resonance is found in any category and the exclusion limit is shown in Figure 9b. 


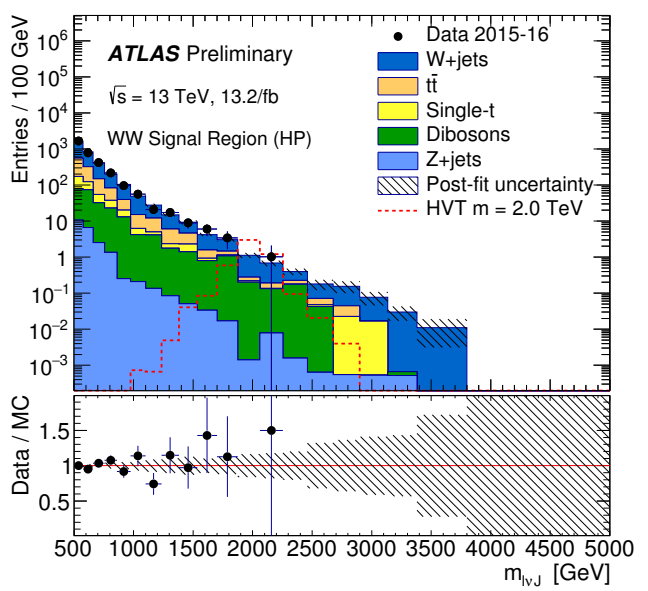

(a) $l v q q^{\prime}$ invariant mass for the merged category, after reconstruction of the neutrino longitudinal momentum based on the $W$ mass constraint.

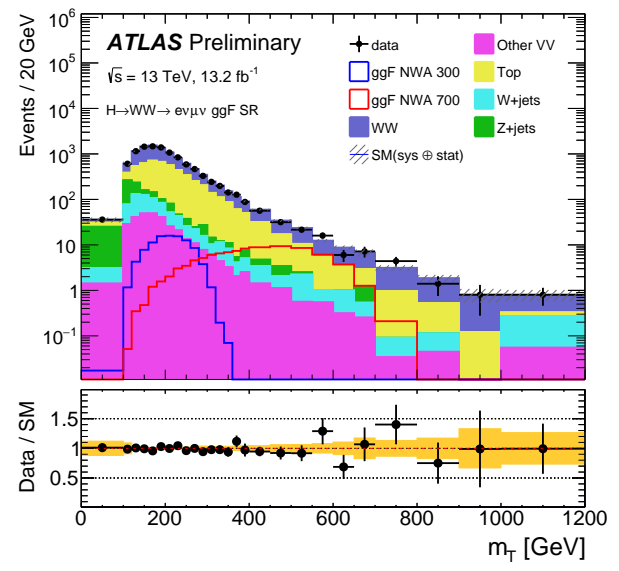

(b) Transverse mass of di-lepton and $\mathrm{E}_{\mathrm{T}}^{\mathrm{miss}}$

Figure 5: Final discriminant variables in searches for a heavy Higgs boson decaying into $l v q q^{\prime}$ and $l v l v$. Plot 5a from Ref. [15] and plot 5b from Ref. [16].

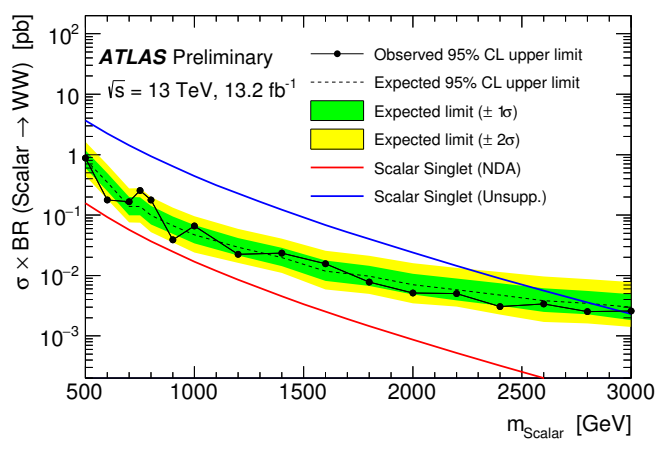

(a) Exclusion limits of the $l v q q^{\prime}$ channel for signals with a narrow width.

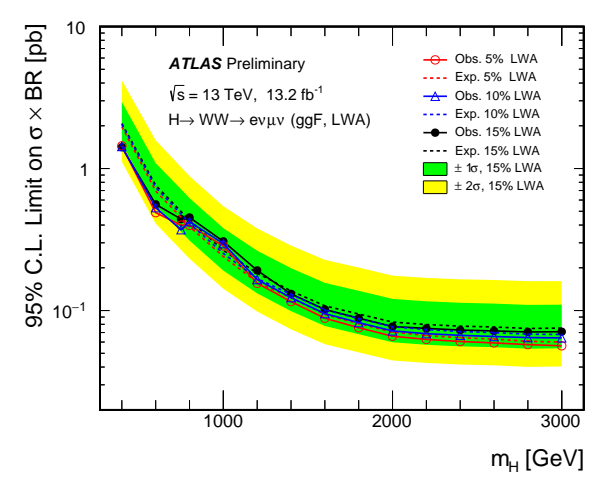

(b) Exclusion limit of the $l v l v$ channel for signals with $5 \%, 10 \%$ and $15 \%$ relative width. The $\pm 1 \sigma$ and $\pm 2 \sigma$ uncertainty bands are for the $15 \%$ relative width.

Figure 6: Exclusion limit in searches for a heavy Higgs decaying into $W W$. Plot 6a from Ref. [15] and plot $6 \mathrm{~b}$ from Ref. [16]. 


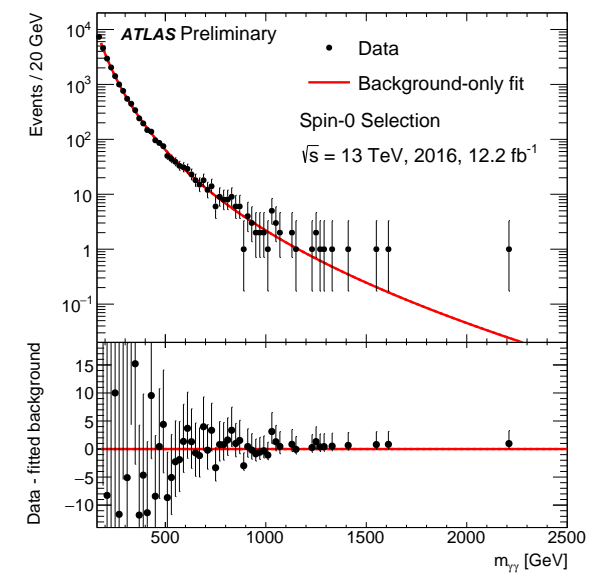

(a) Di-photon invariant mass spectrum of 2016 data.

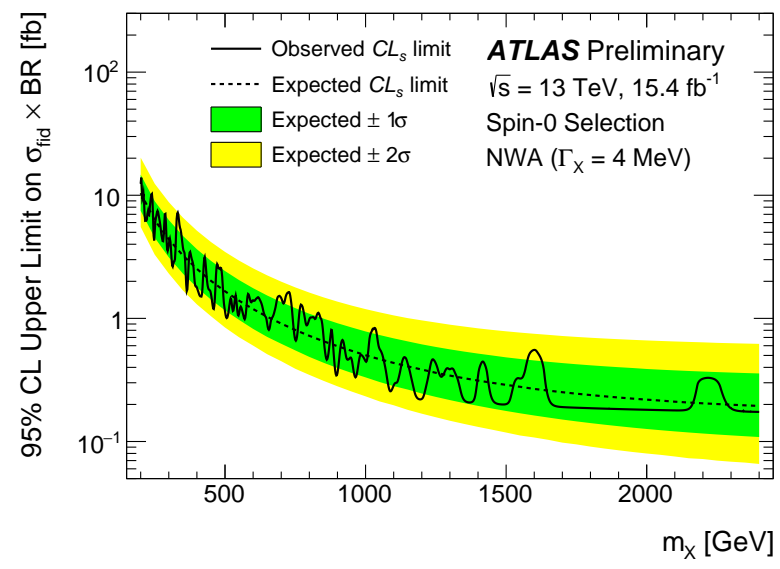

(b) Exclusion limit on a heavy resonance decaying into two photons.

Figure 7: Search for a heavy Higgs boson decaying into di-photon. Plots from Ref. [19].

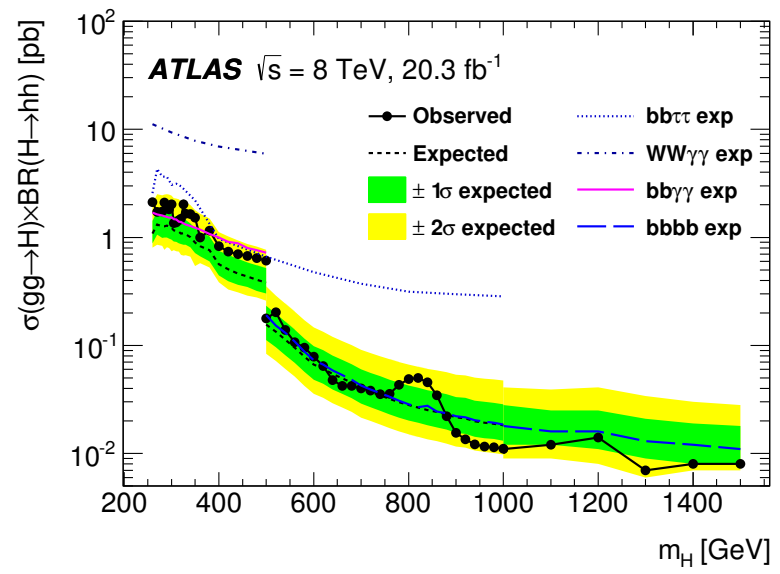

Figure 8: Model independent limits of $\sigma(g g \rightarrow H) \times B R(H \rightarrow h h)$ at $\sqrt{s}=8 \mathrm{TeV}$ as a function of the heavy Higgs boson mass $m_{H}$, combining resonant searches in $h h \rightarrow \gamma \gamma b b, b b b b, b b \tau \tau$ and $\gamma \gamma W W^{*}$ final states. Plot from Ref. [20].

\section{Summary}

A number of searches for neutral BSM Higgs boson have been conducted by ATLAS with the newly collected LHC $13 \mathrm{TeV}$ data in various event topologies. No significant hint of BSM physics has been found so far. However, tighter constraints have been put on BSM models, e.g. 2HDM, MSSM, which will help to find the next path for the new physics search. With more than $30.0 \mathrm{fb}^{-1}$ data by the end of 2016 , more exciting results are expected.

\section{References}

[1] ATLAS Collaboration, Observation of a new particle in the search for the Standard Model Higgs boson with the ATLAS detector at the LHC. Physics Letters B 716 (2012) 1-29. 


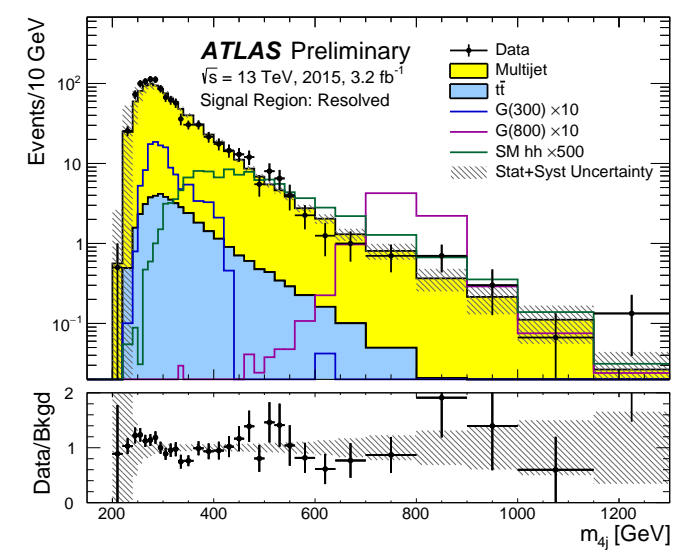

(a) $4 b$ invariant mass in the resolved category.

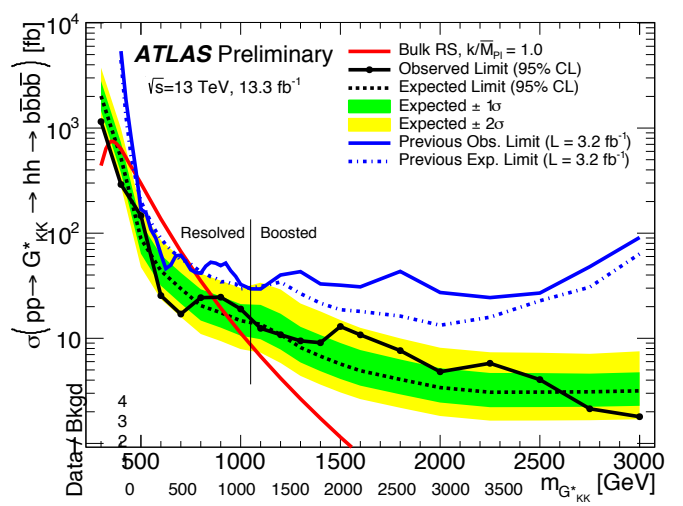

(b) Exclusion limit for a heavy resonance decaying into $b b b b$.

Figure 9: Search for a heavy resonance decaying into a Higgs boson pair. Plots from Ref. [21].

[2] CMS Collaboration, Observation of a new boson at a mass of $125 \mathrm{GeV}$ with the CMS experiment at the LHC. Physics Letters B 716 (2012) 30-61.

[3] ATLAS Collaboration, The ATLAS Experiment at the CERN Large Hadron Collider. 2008 JINST 3 S08003.

[4] ATLAS Collaboration, Constraints on new phenomena via Higgs boson couplings and invisible decays with the ATLAS detector. Journal of High Energy Physics 11 (2015) 206.

[5] ATLAS Collaboration, Searches for Higgs boson pair production in the $h h \rightarrow b b \tau \tau, \gamma \gamma W W^{*}$, $\gamma \gamma b b$, bbbb channels with the ATLAS detector. Phys. Rev. D92 (2015) 092004. https://atlas.web.cern. ch/Atlas/GROUPS / PHYSICS/PAPERS/HIGG-2013-33/

[6] ATLAS Collaboration, Search for the Minimal Supersymmetric Standard Model Higgs bosons H/A in the $\tau \tau$ final state in up to $13.3 \mathrm{fb}^{-1}$ of pp collision data at $\sqrt{\mathrm{s}}=13 \mathrm{TeV}$ with the ATLAS detector . http: / / cds. cern. ch/record/2206278 ATLAS-CONF-2016-085.

[7] ATLAS Collaboration, A search for tt resonances using lepton-plus-jets events in proton-proton collisions at $\sqrt{s}=8 \mathrm{TeV}$ with the ATLAS detector. Journal of High Energy Physics 08 (2015) 148.

[8] ATLAS Collaboration, Search for heavy Higgs bosons A/H decaying to a top-quark pair in pp collisions at $\sqrt{s}=8 \mathrm{TeV}$ with the ATLAS detector. http: / / cds. cern. ch/record/2206229 ATLAS-CONF-2016-073.

[9] ATLAS Collaboration, Search for high-mass diboson resonances with boson-tagged jets in proton-proton collisions at $\sqrt{s}=8 \mathrm{TeV}$ with the ATLAS detector. Journal of High Energy Physics 1512 (2015) 055.

[10] ATLAS Collaboration, Searches for heavy diboson resonances in pp collisions at $\sqrt{s}=13$ TeV with the ATLAS detector. Journal of High Energy Physics 09 (2016) 173.

[11] ATLAS Collaboration, Study of the Higgs boson properties and search for high-mass scalar resonances in the $H \rightarrow Z Z^{*} \rightarrow 4 l$ decay channel at $\sqrt{s}=13$ TeV with the ATLAS detector. https://cds.cern.ch/record/2206253 ATLAS-CONF-2016-079.

[12] ATLAS Collaboration, Searches for heavy ZZ and ZW resonances in the llqq and vvqq final states in pp collisions at $\sqrt{s}=13$ TeV with the ATLAS detector.

http: / / cds. cern. ch/record/2206275 ATLAS-CONF-2016-082. 
[13] Andrew J. Larkoski, Ian Moult, Duff Neill, Power counting to better jet observables. Journal of High Energy Physics 12 (2014) 009.

[14] Andrew J. Larkoski, Ian Moult, Duff Neill, Analytic Boosted Boson Discrimination. https://cds.cern.ch/record/2206253 arXiv:1507.03018.

[15] ATLAS Collaboration, Search for diboson resonance production in the lvqq final state using $p p$ collisions at $\sqrt{s}=13 \mathrm{TeV}$ with the ATLAS detector.

http://cds.cern.ch/record/2206199 ATLAS-CONF-2016-062.

[16] ATLAS Collaboration, Search for a high-mass Higgs boson decaying to a pair of WW bosons in $p p$ collisions at $\sqrt{s}=13 \mathrm{TeV}$ with the ATLAS detector.

http://cds.cern.ch/record/2206243 ATLAS-CONF-2016-074.

[17] ATLAS Collaboration, Search for resonances in diphoton events at $\sqrt{s}=13 \mathrm{TeV}$ with the ATLAS detector. Journal of High Energy Physics 09 (2016) 001

[18] CMS Collaboration, Search for Resonant Production of High-Mass Photon Pairs in Proton-Proton Collisions at $\sqrt{s}=8$ and $13 \mathrm{TeV}$. Phys. Rev. Lett. 117, 051802 (2016).

[19] ATLAS Collaboration, Search for scalar diphoton resonances with $15.4 \mathrm{fb}^{-1}$ of data collected at $\sqrt{s}=13 \mathrm{TeV}$ in 2015 and 2016 with the ATLAS detector . http://cds.cern.ch/record/2206154 ATLAS-CONF-2016-059.

[20] ATLAS Collaboration, Searches for Higgs boson pair production in the $h h \rightarrow b b \tau \tau, \gamma \gamma W W^{*}, \gamma \gamma b b$, bbbb, channels with the ATLAS detector. Phys. Rev. D 92, 092004(2015).

[21] ATLAS Collaboration, Search for pair production of Higgs bosons in the b $\bar{b} b \bar{b}$ final state using proton-proton collisions at $\sqrt{s}=13 \mathrm{TeV}$ with the ATLAS detector.

http://cds. cern. ch/record/2206131 ATLAS-CONF-2016-049. 